\title{
Four Years Comparative Follow-up Evaluation of Community-based, Step-down and Residential Specialist Psychodynamic Programs for Personality Disorders
}

\section{Abstract}

Although the fulcrum of service provision for personality disorder (PD) has shifted from hospital-based to psychodynamically and cognitively-oriented outpatient programs, very few studies have attempted to compare specialist moderate intensity out-patient programs with specialist high intensity residential models, or to explore whether a period of in-patient treatment may be necessary to improve outcome and prognosis. In this article we prospectively compare changes over a 4-year period in three groups of patients with personality disorders $(N=162)$ treated in a specialist community-based (CBP, $N=30)$, a stepdown (RT-CBP, $N=87$ ) and a specialist residential program $(\mathrm{RT}, N=45)$ in psychiatric distress, deliberate self-injury and suicide attempt using multi-level modelling and multivariate logistic regression analyses. The results showed that percentages of early-dropout were significantly different $(p=0.0001)$ for the three programs (CBP $=13.4 \%, \mathrm{RT}-\mathrm{CBP}=10.2 \%$ \& $\mathrm{RT}=41.4 \%)$. A significant interaction between treatment model and time was found for psychiatric distress $(p=0.001)$, with CBP and RT-CBP achieving more marked changes $(g=1.20$ \& $g=0.68$, respectively) compared to RT ( $g=0.30)$ at 48-month follow-up. CBP and RT-CBP were found to significantly reduce impulsive behavior (deliberate self-injury and suicide attempt) compared to RT. Severity of presentation was not found to be a significant predictor of outcome. Long-term RT showed no advantage over long-term CBP, either as stand-alone or as step-down treatment. Replication may be needed to confirm generalizability of results and a number of limitations in the study design may moderate the inferences that can be drawn from the results 


\section{KEY WORDS}

personality disorder, psychodynamic treatment, community-based treatment, residential treatment, outcome study, clinical severity, long-term follow-up

\section{KEY PRACTITIONER MESSAGE}

- Specialist CBP confirmed as effective treatment for PD

- Residential treatment for PD is not indicated unless followed by long-term outpatient treatment

- RT not followed by out-patient treatment was associated with high drop-out and inferior improvement in psychiatric distress and self-harming behavior

- No added benefit of residential treatment was found

- Clinical severity of presentation was not a predictor of long-term outcome 


\section{BACKGROUND}

Over the last three decades a number of specialist approaches for the treatment and management of patients with personality disorder (PD) have been developed. These dedicated treatment programs, based on behavioral, psychodynamic, systemic and cognitive theoretical models have been consistently found to be clinically and cost effective following several randomized and observational studies (Bales et al., 2015; Anthony Bateman \& Fonagy, 2009; Clarkin, Levy, Lenzenweger, \& Kernberg, 2007; Davidson, Tyrer, Norrie, Palmer, \& Tyrer, 2010; Linehan et al., 2006). These approaches have been, for the most part, applied in outpatient settings and have emerged as a less costly alternatives to specialist in-patient and day-patient treatment programs without apparent loss of clinical effectiveness (A. Bateman \& Fonagy, 2010; Giesen-Bloo et al., 2006; Gullestad et al., 2012). In addition, recent reports have highlighted the clinical effectiveness of treatment programs of moderate intensity compared to more intensive programs (Arnevik et al., 2009; Chiesa, Fonagy, \& Gordon, 2009), which may even have adverse effects for patients with severe PD (Chiesa, Fonagy, \& Holmes, 2003; Davidson \& Tran, 2014).

The number of high-intensity long-term residential programs (RT) for PD have declined because the high cost of these was not matched by clear evidence of additional clinical benefit (Chiesa, 2005b; Rutter \& Tyrer, 2003; Schimmel, 1997). However, recent studies have argued that the demise of the RT approach to PD may be premature and that an intensive period of hospital-based treatment may be necessary to tackle the severity of functional impairment, self-harming behaviors presented by some of these patients, and to increase the compliance and response to subsequent outpatient treatment (Bartak, Andrea, 
Spreeuwenberg, Ziegler, et al., 2011; Bohus et al., 2004; Prunetti, Bosio, Bateni, \& Liotti, 2013).

Although outpatient and residential models have been separately evaluated in a number of studies, to our knowledge only one study has directly compared their relative clinical effectiveness (Bartak, Andrea, Spreeuwenberg, Thunnissen, et al., 2011; Bartak, Andrea, Spreeuwenberg, Ziegler, et al., 2011; Bartak et al., 2010). This multi-center non-randomized study found that on average patients treated in specialist in-patient, day-patient and outpatient settings significantly improved over an 18-month period, but a marginal significance was only found in favor of short-term in-patient treatment for Cluster B \& C PD. The very low intensity (one session per week) model employed for out-patient treatment affects the inferences that can be drawn from this comparison.

The available literature on the impact of baseline clinical severity on outcome is limited. Remission rates in BPD were found to be higher in patients with no history of childhood sexual abuse and no family history of substance use disorder (M C. Zanarini, Frankenburg, Hennen, Reich, \& Silk, 2006; M. C. Zanarini, Frankenburg, Hennen, Reich, \& Silk, 2005), while a recent study found that a specialist approach (Mentalization Based Treatment) did better than structured general psychiatric management in a BPD sample with more severe features, such as a higher number of co-morbid PD (A. Bateman \& Fonagy, 2013). A recent studies found that higher severity of presentation predicted lower 2-year recovery rates in a group of borderline PD (M. C. Zanarini et al., 2014) and poorer prognosis (De Panfilis et al., 2011). Another study found that high hostility, childhood abuse, and in-session dissociation were associated with discontinuation of treatment and lower chances of recovery (Arntz, 
Stupar-Rutenfrans, Bloo, van Dyck, \& Spinhoven, 2015). A key clinical indication for RT treatment for PD has been the severity of presenting symptoms and self-harming behaviors (Fenton \& McGlashan, 1990; Norton \& Hinshelwood, 1996). However, there is scant empirical evidence in support of severity as a moderator of outcome for both residential and non-residential treatment.

The current study builds upon previous published work from the same group of researchers regarding the effectiveness and cost-effectiveness of the psychosocial programs offered by the Cassel Hospital, Richmond, United Kingdom, a state-funded tertiary center specialized in the treatment of personality disorder (Beecham, Sleed, Knapp, Chiesa, \& Drahorad, 2006; Chiesa \& Fonagy, 2000; Chiesa \& Fonagy, 2007; Chiesa, Fonagy, Holmes, \& Drahorad, 2004). Although there is a degree of samples overlap with previous reports, for the first time we present results of a comparison of the groups of patients treated in the three hospital treatment programs, including data from patients directly admitted to the specialist longterm community-based program without going through a period of residential stay. In addition, the severity of clinical presentation as moderator of outcome is examined.

The primary aim of the current prospective naturalistic study was to evaluate the clinical effectiveness of the three specialist programs offered to a PD population: a) the communitybased program (CBP) in which patients are admitted directly to the outpatient program; b) the step-down program (RT-CBP) in which patients spend 6 months of residential treatment before stepping down to $\mathrm{CBP} ; \mathrm{c}$ ) the residential only program (RT) in which patients are admitted for a 12-month stay. The three samples were followed-up over a 48-month period and evaluated with regard to level of treatment compliance, psychiatric distress, deliberate 
self-injury and suicide attempt. Our hypothesis was of no significant difference between the three groups. The secondary aims were to investigate the impact of baseline clinical severity on outcome through the identification of possible interactions between clinical severity, outcome and treatment program. We hypothesized that more severe patients responded better to the more intensive conditions offered by RT, based on previous clinically-based assumptions (Gabbard et al., 1999; Norton \& Hinshelwood, 1996).

We hope that the results of this study may provide some evidence about differences in outcome between high intensity/low volume/high cost residential programs and moderate intensity/high volume/low cost out-patient programs that might inform clinically-based decisions with regard to referral pathways for PD.

\section{METHOD}

\section{Treatment programs}

Historically, the Cassel Hospital provided personality disorder (PD) patients with long-term (>1-year stay) residential treatment (RT) based on psychodynamic, psychosocial and therapeutic community principles (Griffiths \& Pringle, 1997; Hinshelwood \& Skogstad, 1998). Patients were discharged back to their own communities with no further planned follow-on treatment. When a 3 to 6 month clinical follow-up assessment was introduced, it came to light that relapses were more frequent than expected, which was thought to be due to a lack of planned follow-on treatment after the sudden discharge into the external community. Therefore, in 1991 a step-down program (RT-CBP) was introduced, which consisted of a RT of reduced length ( 6 months) followed by a long-term ( 2 years) psychodynamic community-based program provided by the same hospital, in order to 
ensure continuity and support after patients returned to community life (Chiesa, 1997;

Chiesa \& Fonagy, 2002). In 2005 the hospital started to admit patients directly to the community-based program (CBP) without going through the RT stage, in order to diversify the services provided and to respond to local referrers' need for an out-patient specialist service for PD. The changes in treatment patterns also reflected administrative changes in the funding of the service. For a more detailed outline of the historical changes in service provision at the Cassel, see Chiesa (2005a) and Chiesa \& Healy (2009).

In the period the research took place, all referrals were sent to the two senior psychiatrists in charge of the in-patient and out-patient teams for an initial assessment. The residential and community-based staff teams are separate and referrals are managed separately. Patients referred specifically for in-patient treatment are allocated to one of the two clinical programs, according to established geographical criteria: patients referred from outside the Greater London area (GLA), who would be unable to continue treatment as out-patients at the Cassel, are allocated to the 1-year stay RT, while patients from within the GLA are admitted to RT-CBT. Patients referred for specialist out-patient treatment are assessed before being allocated to the CBT. A degree of crossover between RT-CBT and CBT (<3\%) following the initial assessment may take place based on clinical considerations.

\section{Community-based psychosocial program (CBP)}

The multidisciplinary clinical team includes a senior psychiatrist, two group psychotherapists, two outreach psychosocial nurses, a nurse supervisor, a social worker and one assistant psychologist. The clinical components of the program consist of an initial psychiatric, psychosocial and psychometric assessment; twice weekly small group psychodynamically-oriented psychotherapy; twice weekly outreach psychosocial nursing 
activities in the community; regular psychiatric reviews by the senior psychiatrist; family and couple therapy as required; social worker's support; multidisciplinary staff meetings once a week; psychotropic medication as required. The initial clinical contract with patients stipulates a 2-year stay, but in most cases a 6 to 12 month extension may be agreed on clinical grounds to consolidate and increase clinical improvement. This is negotiated during the last clinical review with the senior psychiatrist and agreed after team discussion. At termination, patients are either referred back to their original general mental health teams for further care if needed or to their primary care physicians. In addition, patients are invited to a follow-up consultation 3 months after discharge from CBP to discuss possible further psychotherapy treatment outside the Cassel if required and indicated. Up to 28 patients are treated at any one time.

\section{Residential program (RT)}

This is the Cassel traditional multi-component program that consists of a combination of formal psychodynamically-oriented psychotherapy and therapeutic community principles and activities. The psychotherapy includes twice-weekly individual psychotherapy and twice-weekly group psychotherapy. Therapeutic community treatment is supervised by psychosocial nursing staff and includes community meetings five times a week and a program of daily activities aimed at the acquisition of interpersonal skills, re-socialization and rehabilitation. Most patients are on psychotropic medication, which is monitored by psychiatric staff. Formal review of progress occurs as part of CPA multi-disciplinary meetings every two to three months. Staff meetings occurs daily to discuss individual patients and inform case-management. The hospital has an 'open door policy' and all patients are admitted to the hospital on a voluntary basis. Although patients are encouraged to return to their residences for weekends if the clinical situation allows, they are not to leave the 
hospital premises unless previously negotiated with staff. The mental health personnel in the residential unit include 1 senior psychiatrist, 2 senior nurses, 4 junior nurses, 3 individual and 1 group psychotherapists, 2 staff psychiatrists in specialist training and 1 social worker. The unit has a 16-bed capacity. After discharge from RT patients are referred back to their mental health resource centers, which were involved in CPA meetings during the patients RT.

Step-down program (RT-CBP)

Patients are treated for 6 months in the therapeutic community residential setting (as above) before stepping down to the CBP (as above) for a further period of 2-year specialist out-patient treatment. Patients know at initial clinical assessment that they are allocated to the RT-CBP. Although the length of residential stay is set in advance, on the basis of clinical need patients may obtain an extension of up to $6 / 7$ week duration before stepping-down to CBP.

\section{Samples and procedure}

Sample size was calculated on the basis of a fixed effect ANCOVA with 3 groups and a single covariate, including main effect and interaction for a medium effect size and alpha set at 0.05 yielding power at $85 \%$. Medium effect size and alpha set at .05 with a sample size of 164 yields an observed power of $81 \%$ for a one-way omnibus fixed effect ANOVA.

Data was routinely collected from patients admitted to the Hospital three clinical programs, and completed assessment evaluations at intake into treatment, 6, 12, 24, 36 and 48 months. Patients in the CBP were recruited between 2005 and 2011, and followed up until 2016, patients in the RT-CBP were recruited between 1993 and 2011, and followed up until 
2016, and patients in the RT were recruited between 1993 and1998, and followed up until 2002.

All admitted patients were approached within one week of admission to obtain written informed consent to study participation. A CONSORT type diagram shows attrition from the study and from treatment for each program over the 4-year period (figure 1). Of the 250 patients admitted to one of the three treatment conditions (CBP N=43, RT-CBP N=108, RT $\mathrm{N}=99$ ), 25 did not consent to join the study (CBP N=3, RT-CBP N=5 \& RT N=17) and 12 withdrew soon after consenting before intake measures were applied (CBP N=2, RT-CBP N=2 \& RT $N=8$ ). Therefore, the intake study sample comprised of 213 patients (CBP $N=38, R T-C B P$ $\mathrm{N}=102 \& \mathrm{RT} \mathrm{N}=74)$. Of these, a total of 51 dropped-out of the study by 48-month follow-up (CBP N=8, RT-CBP N=14, RT N=29). Thus, a total of 162 patients for which we had at least three assessment points ( $C B P N=30, R T-C B P N=87, R T N=45$ ) were entered into the final comparative analyses. Since the 51 cases that dropped-out of the study protocol withdrew their consent to use the data, we did not included them in the final analyses.

Ethical approval was granted by the Riverside Mental Health Trust for the period 1993-2004, while the protocol for continuing routine data collection for the RT-CBP and CBP samples (2005-2015) was approved by the West London Mental Health Trust R \& D Department. Table 1 outlines the demographic, Axis-II diagnostic, risk factors and severity profiles for the CBP, RT-CBT and RT samples. Patients were well matched in all variables, apart from age and severity of psychiatric distress, due to subjects in the CBP being significantly older and having higher intake GSI scores than subjects in the other two samples. However, there was 
no significant difference between the three groups in overall clinical severity of presentation $\left(\chi^{2}(2)=0.87, p=0.647\right)$, defined as the median number of Axis-II diagnoses each patient met criteria for, number of positive PD criteria met and intake psychiatric distress scores.

The average age for the whole sample was 34.1 ( $S D=8.9$, median=32.0, range 19-60), the majority were female (77.2\%), single (67.9\%), college educated $(51.9 \%)$ and unemployed (81.4\%). The average number of PD patients met criteria for was 3.65 ( $S D=1.89$, median=3.00, range $1-10$ ), with $87 \%$ of the sample meeting criteria for more than one PD diagnosis. Borderline PD, avoidant PD, dependent PD, depressive PD and paranoid PD were the most frequent Axis-II diagnoses. Patients reported at interview high rates of early loss (46.9\%), sexual abuse (50.6\%) and physical abuse (54.3\%). All patients presented with a high level of severity and chronicity with regard to self-harming behavior, suicidality, mental health service utilization, psychiatric distress, substance abuse and interpersonal and social dysfunction. Most patients presented with a long list of ineffective previous treatment attempts by mental health professionals.

\section{Measures}

The Structured Clinical Interview for DSM-IV-II (SCID-II) (First, Gibbon, Spitzer, Williams, \& Benjamin, 1997) was applied to each participant for identification of Axis-II Personality Disorders. The SCID-II is based on the diagnostic criteria of the Diagnostic and Statistical Manual for mental disorders version IV (American Psychiatric Association, 1995).

The Brief Symptom Inventory (BSI) is a 53-item, self-rated on a 5-point scale test that measures patients' subjective experience of psychiatric symptoms in nine different areas 
(somatization, obsessive-compulsion, interpersonal sensitivity, depression, anxiety, psychoticism, phobic anxiety, hostility and paranoid ideation). The Global Severity Index (GSI), calculated by dividing the total score by the number of items in the BSI, is the general score used in this study to report changes in the dimension of psychiatric distress.

The Cassel Community Adjustment Questionnaire (Chiesa et al., 2004), a structured interview modelled on the Suicide and Self-Harm Inventory (A Bateman \& Fonagy, 1999), collected details of deliberate self-injury, attempted suicide episodes over the year prior to the assessment and at each assessment point for the previous year. Each variable was operationally defined and the interviewer was required to write details that would be checked for accuracy by a senior psychiatrist (MC). Deliberate self-injury was defined as deliberate injury inflicted to one's own body tissue in the absence of intent to die, including cutting, burning, scratching, banging and biting. Suicide attempt was defined as a non-fatal self-directed behavior with an intent to die as a result of that behavior. A random sample of the interviews was cross-checked against the records of the patients' general practitioners (median $K=0.88$, range $=0.76-1.00$ ), and a second sample was subjected to test-retest reliability checks (median $K=0.84$, range=0.73-0.96).

\section{Data analyses}

All analyses were performed using the Statistical Package for the Social Sciences (SPSS for windows) version 22. The prospective analyses of changes for psychiatric distress (GSI), deliberate self-injury (NSSI) and suicide attempt were set at the beginning of the study. Comparison of reliable change and clinical significant change between the groups and evaluation of the predictive significance of clinical severity on outcome were post-hoc analyses. 
In order to test baseline differences in the three samples' demographic, diagnostic and risk factors variables, Pearson chi square statistics with 95\% confidence intervals (Cls) for the odds ratios for categorical variables, and t-tests for independent samples with $95 \% \mathrm{Cls}$ for the difference in mean scores for continuous variables were used.

In order to compare differences in outcome between the three specialist programs, we investigated levels of change over time in the primary outcome measure (BSI-GSI) and evaluated the presence of difference between groups over the 4-year period, by using multi-level modelling (MLM) (Field, 2013; Rabe-Hesketh \& Skrondal, 2008). The model was iteratively built by initially including GSI as the dependent variable, and Time as covariate. Allowing the intercept to vary randomly significantly improved the model fit $\left(\chi^{2}(1)\right.$ change $=339.75, p=0.0001)$. Specifying both intercept and slopes as random for the effect of Time, further improved the fit of the model $\left(\chi^{2}(2)\right.$ change $\left.=44.40, p=0.001\right)$. After testing four different covariance structures to the model, covariance as 'Unstructured' yielded the best fit to the data. We then added a further covariate 'Treatment Year', which was found to positively correlate with intake GSI $(r=0.26, p=0.001)$, before adding into the model the independent variable and its interactions with the covariates.

In order to test the impact of clinical severity of presentation on outcome, we divided each sample into a severe and (relatively) less severe sub-groups. Severity was assessed through the aggregation of the following variables at baseline: number of Axis-II diagnoses each patient met criteria for, number of positive PD criteria met and intake psychiatric distress scores as indicated by the Brief Symptoms Inventory general severity index (GSI). The 
sample median was used as a cut-off point for all severity variables - three or more PD diagnoses, 30 or more positive PD criteria met and a score above 2.06 on the GSI. Participants below the median on at least two out of the three indicators of severity were grouped as relatively less severe. These criteria are in line with the assessment of severity features in PD outlined in recent reports as well as both DSM-5 (Section III) and ICD-11 (American Psychiatric Association, 2013; A. Bateman \& Fonagy, 2013; Crawford, Koldobsky, Mulder, \& Tyrer, 2011; Tyrer, Reed, \& Crawford, 2015). A MLM model with GSI as the dependent variable, treatment group as fixed factor, and time and clinical severity at intake as covariates, with random intercept and slope, was performed to test the significance of the 3-way interaction between these factors. The attempt to combine these measures into a single continuous variable using standardized scores, yielded a low index of consistency $(\alpha<0.7)$. However, we looked at the significance of psychiatric distress at intake on the MLM.

In order to evaluate the clinical relevance of the changes in GSI over time, we calculated the average pre/post effect size (Hedge's g) for each sample on the predicted means at each assessment point. Hedge's g was calculated by subtracting the post-test mean to the pretest mean divided by the pooled and weighted standard deviation (mean post $_{-}-$mean $_{\text {pre }}$ ) $S D_{\text {pooled }}$ (Ellis, 2010). Outcome was also analyzed as categorical variables by calculating reliable change indexes $(\mathrm{RCI})$ and rates of clinical significant change (CSC) for GSI using the formulas devised by Jacobson and Truax (Jacobson \& Truax, 1991). RC refers to changes that are not attributable to measurement error; it is derived by subtracting the post-test mean to the pre-test mean divided by the standard error of the difference $\left(\right.$ mean $_{\text {post }}-$ mean $_{\text {pre }} / S E_{\text {diff, }}$ where $\left.S E_{\text {diff }}=S D_{\text {pre }} * \sqrt{ } 2 * \sqrt{ } 1-r x x\right)$. Change that exceed 1.96 times the value of $S E_{\text {diff }}$ are 
deemed to constitute reliable improvement or deterioration according to the direction of the change. In order to calculate CSC, we used Truax \& Jacobson criterion C, which requires that a patient falls in the normative distribution than a clinical sample distribution after the treatment intervention. Therefore, we obtained a GSI distribution (mean=0.68, SD=0.44) from a non-psychiatric sample $(n=122)$, matched to our PD sample for age and gender, recruited from medical and surgical departments of a metropolitan general hospital (UCLH). Separate cut-off points were calculated for each PD sample according to the formula cutoff $=\left(S D_{\text {normal sample }}{ }^{*}\right.$ mean pre $P D$ sample $)+\left(S D_{\text {pre } P D \text { sample }} *\right.$ mean normal sample $) /\left(S D_{\text {normal }}\right.$ sample $+S D_{\text {pre }}$ PD sample) .4 patients in $\mathrm{CBP}, 10$ patients in RT-CBP and 9 patients in RT were found to have intake GSI already below the cut-off point for CSC and were excluded from the analysis. Kendal's Tau-b statistics and Pearson chi square for ordered contingency tables were used to test the significance of the differences between groups in RC and CSC, respectively. Since differences in intake GSI scores may affect comparisons between the three samples (Evans, Margison, \& Barkham, 1998), separate multinomial regression and logistic regression analyses with reliable change at each assessment point as the dependent variable, group status as predictor variable and intake GSI as covariate, were carried out to test whether treatment group was a significant predictor of improvement and recovery.

In order to test differences of proportions for deliberate self-injury and attempted suicide between the groups, separate logistic regressions for binary data were used, with presence or absence of self-injury or attempted suicide at $12,24,36$ and 48 month follow-up as the dependent variable and treatment status, attempted suicide/self-injury status at intake and treatment year as predictors. 
Since multiple testing may inflate the likelihood of false positive findings, the significance threshold in all analyses pertaining to outcome was set at 0.01 .

\section{RESULTS}

\section{Length of treatment, drop-out and suicide}

The average duration of treatment for the $\mathrm{CBP}$ intake study sample $(\mathrm{N}=38)$ was 26.3 months $(\mathrm{SD}=13.8$, median=30.0, range=0.7-46.0), for the $\mathrm{RT}-\mathrm{CBP}$ intake sample $(\mathrm{N}=101)$ was 23.8 months ( $S D=11.0$, median=22.4, range $=0.9-46.2)$ and for the $R T$ intake sample $(N=74)$ was 6.7 months $(S D=4.4$, median $=6.5$, range $=0.3-13.5)$. Figure 2 outlines the percentage of treatment attrition over time in the three programs.

Since the three programs have different treatment lengths, we used a somewhat arbitrary 5-months cut-off point to compare differences in percentages of early drop-out between the three programs. Drop-out was defined as the premature termination of treatment either by patients unilaterally leaving the programs or by staff discharging patients from the program due to dangerous and repeated acting-out behavior or lack of engagement with the programs. We examined levels of early drop-out in all patients admitted to the programs, and we found that 33 (86.6\%) CBP patients, 97 (89.8\%) RT-CBP patients and 58 (58.6\%) were still in treatment after 5 months from admission. The difference in early dropout between the three groups was highly significant $\left(\chi^{2}(2)=41.70, p=0.0001\right)$. The odds ratio for dropout by 5 months for patients in RT was 5.4 (95\% Cl 1.9-14.9) compared to CBP and $6.2(95 \% \mathrm{Cl} 3.0-13.1)$ compared to RT-CBP. It is to note that a number ( $\mathrm{N}=17,16.8 \%)$ of $\mathrm{RT}-$ CBP patients ended prematurely the in-patient stage of treatment, but agreed to continue treatment in $\mathrm{CBP}$ after discharge, hence they were not regarded as drop-out 
No patients in CBP committed suicide, one patient killed herself in RT-CBP and 5 patients committed suicide in RT. Of the successful suicides, only 2 patients in RT were still in active treatment at the time the tragic event took place.

\section{Psychiatric distress and severity}

The main model yielded a significant linear relationship between GSI and time $\left(F_{(1,156.7}=7.28\right.$, $p=0.008)$, indicating that the samples improved significantly over the 4-year period, and a significant interaction between treatment model and time $\left(F_{(2,157.5)}=7.04, p=0.001\right)$. As Figure 3 shows, the linear trend of the GSI decline was significantly greater in CBP compared

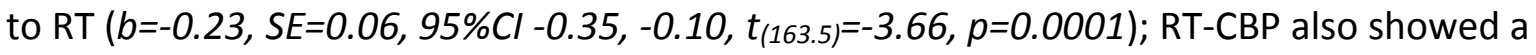
significant greater decline in the slope compared to RT $(b=-0.11, S E=0.04,95 \% \mathrm{Cl}-0.18,0.03$, $\left.t_{(136.1)}=-2.87, p=0.005\right)$. Treatment year was significant covariate in the model $\left(F_{(1,160.9)}=5.09\right.$, $p=0.025)$, which significantly interacted with time $\left(F_{(1,156.8}=7.20, p=0.008\right)$. The positive value of the interaction between treatment year and time (Table 2) indicates that in later treatment year the decrease in observed GSI over time was less marked. There was no significant association between treatment year and severity of presentation $\left(B_{(1)}=-0.02\right.$, $S E=0.03, p=0.657)$, suggesting no change in severity of presentation by patients admitted over the years the study took place.

The measure of the magnitude of the improvement in the three groups expressed as effect size (Hedge's $g$ ) from intake through to 48 months follow-up, showed that the CBP achieved a small effect $(g=0.28)$ by 6 months, a moderate effect $(g=0.55)$ by 12 months and increasingly large effects at 24 months $(g=0.80), 36$ months $(g=1.03)$ and 48 months ( $g=1.23)$. By comparison, RT-CBP showed small effect sizes at 6,12 and 24 months $(g=0.17$, 
$g=0.32$ and $g=0.47$, respectively), and reached moderate effects at 36 and 48 months ( $g=0.58$ and $g=0.68$ ). Finally, RT obtained small effects: $g=0.07$ at 6 months, $g=0.14$ at 12 months, $g=0.20$ at 24 months, $g=0.26$ at 36 months and $g=0.30$ at 48 months.

No significant interaction between Time and baseline severity $\left(F_{(1,151.1)}=1.64, p=0.202\right)$ or between Time, severity and treatment group $\left(F_{(2,166.8)}=1.88, p=0.156\right)$ in $\mathrm{GSI}$ change was found in the MLM, indicating that severity of presentation was not a significant predictor of outcome, both overall and specific to the treatment model (Figure 4). For the severe subsample, improvement expressed in effect size was more marked in CBP ( $g=1.34)$, while RTCBP and RT achieved a moderate effect size ( $g=0.64$ and $g=0.55$, respectively). With less severe patients CBP $(g=1.14)$ and RT-CBP ( $g=0.87)$ obtained a large effect size, while no change was observable for RT ( $g=0.07)$. We also tested the effect of initial severity of psychiatric distress (GSI) as a continuous measure of severity on outcome, and found that the covariance between GSI intercept and slopes in the MLM was not significant $(b=-.02$, $S E=0.01, Z=-1.84,95 \% C l-.04,0.00, p=0.066)$.

The number and percentage of patients who achieved reliable change (improved) and clinically significant change (recovered) are displayed in Figure 5. Patients in the CBP showed progressively high percentages of reliable change at each of the five assessment points, from $50 \%$ at 6 months to $71 \%$ at 48 month follow-up; RT-CBP patients increased percentages of improvement from $36 \%$ at 6 months to $61 \%$ at 48 months; and RT patients showed proportions of improvement from $29 \%$ after 6 months to $43 \%$ after 48 months, with a peak of $61 \%$ at 36 months. Levels of reliable deterioration was lowest in the CBP group 
(ranging from a highest of $20 \%$ at 12 months to a lowest of $4 \%$ at 24 and 48 months), highest in RT-CBP (ranging from a highest of $43 \%$ at 12 months to a lowest of $20 \%$ at 48 months), while RT ranged from a highest of $24 \%$ at 24 months to a lowest of $17 \%$ at 48 months. The differences between the three groups almost reached significance at 24 (Kendall's tau- $b=-0.15, p=0.018$ ) and 48 months (Kendall's tau- $b=-0.17, p=0.017$ ). The results of the likelihood ratio tests in the multinomial logistic regression analyses controlling for intake levels of GSI, showed that group status was a significant predictor of RC at 12 months $\left(\chi^{2}(4)=19.09, p=0.001\right)$ and 24 months $\left(\chi^{2}(4)=20.94, p=0.000\right)$. RT-CBP was found to have higher percentages of clinically significant change at 6 months (21.3\%) compared to CBP (0.0\%) and RT (0.0\%), 12 months (29.6\%) compared to CBP (12.0\%) and RT (2.7\%), 24 months (35.1\%) compared to CBP (12.5\%) and RT (5.4\%), 36 months (34.6\%) compared to CBP (19.0\%) and RT (13.9\%) and 48 months (37.1\%) compared to CBP (22.7\%) and RT (17.6\%). The difference between groups was significant at 6, 12 and 24 months $\left(\chi^{2}{ }_{(2)}=15.19, p=0.001, \chi^{2}{ }_{(2)}=12.08, p=0.002\right.$ and $\chi^{2}{ }_{(2)}=14.21, p=0.001$, respectively). Group status was found to be a near-significant predictor of CSC, controlling for intake levels of GSI, at 12 months $\left(\right.$ Wald $_{(2)}=7.41, p=0.025$, Nagelkerke $\left.R^{2}=0.18\right)$ and 24 months $\left(\right.$ Wald $_{(2)}=8.65, p=0.013$, Nagelkerke $\left.R^{2}=0.23\right)$.

\section{Deliberate self-injury and suicide attempts}

Number and percentages for deliberate self-injury, suicide attempt and psychiatric readmission with odds ratio and 95\% confidence intervals for the three samples are displayed in table 3.

The logistic regression analyses controlling for treatment year and intake levels of NSSI revealed that treatment group was a significant predictor for deliberate self-injury (NSSI) at 
12-months ( Wald $_{(2)}=12.15, p=0.002$, Nagelkerke $\left.R^{2}=0.43\right)$. CBP showed a marked reduction in NSSI compared to RT at 12 months $\left(B_{(1)}=2.47, S E=0.82, p=0.003\right)$ and at 24 months $\left(\mathrm{B}_{(1)}=1.62, \mathrm{SE}=0.81, \mathrm{p}=0.044\right) . \mathrm{RT}-\mathrm{CBP}$ had significantly lower percentages of NSSI compared to RT at 12 months $\left(B_{(1)}=1.81, S E=0.55, p=0.001\right)$, and almost significant reduction at 24 months $\left(B_{(1)}=1.24, S E=0.53, p=0.020\right)$ and 48 months $\left(B_{(1)}=1.17, S E=0.52, p=0.030\right)$. We found no significant differences between CBP and RT-CBP.

With regard to suicide attempt treatment model was a near-to-significance predictor at 12month $\left(\right.$ Wald $_{(2)}=7.38, p=0.025$, Nagelkerke $\left.R^{2}=0.22\right), 36$ months $\left(\right.$ Wald $_{(2)}=7.89, p=0.019$, Nagelkerke $\left.R^{2}=0.16\right)$ and 48-month $\left(\right.$ Wald $_{(2)}=7.19, p=0.027$, Nagelkerke $\left.R^{2}=0.32\right)$. CBP was found to reduce the likelihood of suicide attempt compared to RT at 12 months $\left(B_{(1)}=2.07\right.$, $S E=0.84, p=0.014)$ and 36 months $\left(B_{(1)}=2.63, S E=1.05, p=0.012\right)$. RT-CBT was found to have less suicide attempts compared to RT at 12 months $\left(B_{(1)}=1.22, S E=0.51, p=0.018\right), 36$ months $\left(B_{(1)}=1.97, S E=0.74, p=0.008\right)$ and 48 months $\left(B_{(1)}=2.00, S E=0.75, p=0.007\right)$. No significant differences were found between CBP and RT-CBP.

\section{DISCUSSION}

This is one of few studies that has compared the effectiveness of specialist programs for PD: community-based treatment only, step-down from residential to community-based intervention and residential stay only. The results obtained over a period of four years show that CBP and RT-CBP achieved significantly greater improvement in several dimensions of personality disorder functioning compared to RT. Reduction by 48-month follow-up in general psychiatric distress was particularly marked in CBP $(g=1.23)$, compared to moderate change in RT-CBP $(g=0.68)$, and to modest changes in RT ( $g=0.30)$. CBP and RT-CBP had 
lower odds of engaging in acts of non-suicidal self-injury and attempted suicide at most of the assessment points compared to RT, while we found no difference between CBP and RTCBP. Surprisingly, rates of NSSI increased in the RT sample in the first 12 months from intake, although it gradually decreased during later follow-up assessments.

Before considering the inferences from these results, we would like to outline a number of limitations present in this study that should inform the interpretations of the results. First, the naturalistic nature of the comparison of the samples is a limitation that does not allow to reach definite conclusions with regard to effectiveness. Second, the periods of recruitment was different in each sample raising the possibility that admitted patients' clinical features may have been different between groups and over time. Although it may be argued that referral for residential treatment may indicate a higher level of chronicity and treatment resistance, we showed that groups were well matched on several demographic, diagnostic and risk factor variables, including clinical severity of presentation. This may not be surprising since, in the absence of clear guidelines for referral pathways for personality disorder, it is likely that referral for inpatient or community-based treatment is less influenced by severity of presentation and may depend more on treatment programs availability, cost and referrers' choice (Chiesa, Bateman, Wilberg, \& Friis, 2002). In the light of the finding that severity of admitted patients did not increase over time, the explanation for treatment year being a significant covariate in the model may reside in change in the effectiveness of treatment, once the model of treatment is accounted for (all models of treatment appear to be most effective when they are first introduced) (Fonagy, Luyten, \& Bateman, 2017). Third, the original sample size calculation did not take into account of the high level of attrition present in this study, which consequently is likely to be 
underpowered. However, given the naturalistic nature of the study, multi-level modelling with participants intercept and slope included as random effects is likely to provide a better fit to the data than using a simple fixed effects model. Finally, the difference in baseline level of GSI in the tree groups may affect the interpretation of effect sizes and estimates of Reliable and Clinically Significant Change associated with the law of initial values. However, controlling for GSI at baseline did not substantially affect the results.

Bearing in mind these caveats, our findings suggest that PD patients treated in a specialist community-based model and in a step-down condition based on psychodynamic and psychosocial principles and practices, are more likely to achieve significantly greater improvement in a number of dimensions compared to long-term residential treatment only. The highly significant lower early drop-out rates found in CBP compared to RT, suggests that this treatment model has satisfactory acceptability for PD patients. The significant improvement in self-harming behaviors in the CBP and CBP-RT groups confirm that these programs may be effective in markedly reducing impulsive acting-out behavior on a longterm basis, while the non-significant changes in deliberate self-injury and suicide attempts in RT raise questions about the effectiveness of long-term RT to reduce the risk of self-harm. It is possible that these results can be explained by two considerations. First, CBP and CBPRT patients were treated in a long-term program (median=30 months and 22 months, respectively), while RT patients had treatment for up to one year (median=6.5), albeit in a high intensity program. RT patients were suddenly discharged back to their community of origin and general psychiatric services, without any further follow-up from the specialist treating institution. This lack of continuity of psychodynamically-based treatment may have had a destabilizing effect in several of these patients, who may have felt lost, unsupported 
and abandoned (National Institute for Health and Clinical Excellence, 2009; M. C. Zanarini, 2009). This may have led to regressive phenomena and relapses to maladaptive behaviors. Second, the finding in the RT group of higher early drop-out rates, the increase in nonsuicidal self-injuries, the fairly high rates of reliable deterioration in psychiatric distress at 6 months in both RT and RT-CBP, may suggest the presence of possible iatrogenic effects of residential treatment for a sizeable subgroup of patients, as also remarked by other authors (Davidson \& Tran, 2014; Gunderson, 2001; Paris, 2004). The discrepancy in residential dropout between RT and RT-CBP may be explained by the number of RT-CBP patients (17\%) who discontinued RT stay but continued as CBP patients, and were not considered drop-out. Anecdotally, several patients found having a shorted RT stay and the prospect of joining long-term CBP an incentive for desisting from dropping-out and tolerating the high intensity, pressurizing conditions present in the RT program.

The results of this study add to emerging evidence that specialist high-intensity out-patient programs yield good outcomes similar to those obtained in more intensive partial hospitalization programs and inpatients programs for PD (Arnevik et al., 2010; Gullestad et al., 2012). Similar results have been reported in the treatment of eating disordered populations (Gowers, Weetman, Shore, Hossain, \& Elvins, 2000), which strengthen the 'less may be more' argument in the treatment of non-psychotic disorders (Chiesa et al., 2003). The evidence provided by these studies should be used to inform service delivery and development, with a shift from low-volume/high intensity/high-cost programs to specialist high volume/moderate intensity/lower-cost programs, without loss of clinical effectiveness. The issue of the cost-benefit ratio must be taken into account. Specialist CBP in a UK context is at least six times less expensive to deliver than RT (Chiesa et al., 2009), making it more 
cost-effective (Beecham et al., 2006). In the absence of clear evidence of superior efficacy, we suggest that long-term residential treatment should be either discontinued or confined to cases (e.g. forensic conditions) in which the patient's potential cost-benefit of treatment outweighs the cost-benefit of maintaining the patient in their community, or when an alternative effective treatment is not available.

We also addressed the confounding factor represented by the potential clinical effect of previous specialist inpatient treatment before stepping down to a specialist CBP intervention. We found no evidence that a prior RT stay improves outcome in CBP. CBP only patients had similar improvement rates in psychiatric distress, deliberate self-injury and suicide attempt compared to patient who stepped-down to CBP after a period of residential treatment (RT-CBP). The small effect size $(g=0.11)$ found in GSI obtained by RT-CBP during the 6-month inpatient stage of treatment, indicates that most of the improvement achieved was ascribable to the effects of CBP after discharge from the residential setting. This finding has to be measured against studies that claim, albeit in a qualified fashion, that borderline PD patients may require brief hospital admissions for 'containment' and 'stabilization' (Horn et al., 2015; Leichsenring et al., 2016; Siefert, 2012; Vermote et al., 2015). These studies show that in some settings short-stay inpatient treatments are associated with relatively good outcomes. The differences with our finding may be explained by differences in the treatment model or shorter durations of inpatient stay. The level of severity of the in our CBP sample was higher than in many of the studies reporting positive outcomes for inpatient treatments, which may indicate that, paradoxically, it is severe PD groups who are more likely to experience benefit from specialist CBP. This is consistent with the observation reported by our group (Chiesa, Sharp, \& Fonagy, 2011) that patients who chronically self- 
harmed were most likely to experience iatrogenic effects when treated as inpatients.

However, we emphasize that our results are preliminary and that further controlled studies comparing mixed residential/outpatient models with community-based models are necessary to clarify the extent to which a period of inpatient treatment may or may not be a necessary requirement to improve outcome in this population.

In conclusion, the overall results of this study show evidence of the effectiveness of CBP, which is increasingly becoming the most commonly offered model of care for PD. Long-term RT was shown not to offer advantages over specialist long-term OP. Therefore, the role of long-term RT for personality disorders needs to be reconsidered and redefined, suggesting the need to develop assessment tools for identifying which patients might benefit from an inpatient program and which patient may be harmed by admission to long-term institutional treatment. However, this study does not provide evidence against short-term RT, which might possibly be effective in the treatment of PD. 


\section{References}

American Psychiatric Association. (1995). Diagnostic and Statistical Manual for Mental Disorders (DSM-IV) (IV ed.). Washington: American Psychiatric Press.

American Psychiatric Association. (2013). Diagnostic and Statistical Manual of Mental Disorders (5th ed.). Arlington, VA: American Psychiatric Publishing.

Arnevik, E., Wilberg, T., Urnes, O., Johansen, M., Monsen, J. T., \& Karterud, S. (2009). Psychotherapy for personality disorders: short-term day hospital psychotherapy versus outpatient individual therapy - a randomized controlled study. European Psychiatry, 24, 71-78. doi:10.1016/j.eurpsy.2008.09.004

Arnevik, E., Wilberg, T., Urnes, O., Johansen, M., Monsen, J. T., \& Karterud, S. (2010). Psychotherapy for personality disorders: 18 months' follow-up of the ulleval personality project. J Pers Disord, 24, 188-203. doi:10.1521/pedi.2010.24.2.188

Arntz, A., Stupar-Rutenfrans, S., Bloo, J., van Dyck, R., \& Spinhoven, P. (2015). Prediction of treatment discontinuation and recovery from Borderline Personality Disorder: Results from an RCT comparing Schema Therapy and Transference Focused Psychotherapy. Behav Res Ther, 74, 60-71. doi: $10.1016 /$ j.brat.2015.09.002

Bales, D. L., Timman, R., Andrea, H., Busschbach, J. J. V., Verheul, R., \& Kamphuis, J. H. (2015). Effectiveness of Day Hospital Mentalization-Based Treatment for Patients with Severe Borderline Personality Disorder: A Matched Control Study. Clinical Psychology \& Psychotherapy, 22, 409-417. doi:10.1002/cpp.1914

Bartak, A., Andrea, H., Spreeuwenberg, M. D., Thunnissen, M., Ziegler, U. M., Dekker, J., ... Emmelkamp, P. M. (2011). Patients with cluster a personality disorders in psychotherapy: an effectiveness study. Psychotherapy and Psychosomatics, 80, 88-99. doi:10.1159/000320587

Bartak, A., Andrea, H., Spreeuwenberg, M. D., Ziegler, U. M., Dekker, J., Rossum, B. V., ... Emmelkamp, P. M. (2011). Effectiveness of Outpatient, Day Hospital, and Inpatient Psychotherapeutic Treatment for Patients with Cluster B Personality Disorders. Psychothererapy and Psychosomatics, 80, 28-38. doi: $10.1159 / 000321999$

Bartak, A., Spreeuwenberg, M. D., Andrea, H., Holleman, L., Rijnierse, P., Rossum, B. V., ... Emmelkamp, P. M. G. (2010). Effectiveness of Different Modalities of Psychotherapeutic Treatment for Patients with Cluster C Personality Disorders: Results of a Large Prospective Multicentre Study. Psychotherapy and Psychosomatics, 79, 20-30. doi:10.1159/000254902

Bateman, A., \& Fonagy, P. (1999). Effectiveness of partial hospitalization in the treatment of borderline personality disorder: A randomized controlled trial. American Journal of Psychiatry, 156, 1563-1569. doi:10.1176/ajp.156.10.1563

Bateman, A., \& Fonagy, P. (2009). Randomized Controlled Trial of Outpatient Mentalization-Based Treatment Versus Structured Clinical Management for Borderline Personality Disorder. American Journal of Psychiatry, 166, 1355-1364. doi:10.1176/appi.ajp.2009.09040539

Bateman, A., \& Fonagy, P. (2010). Mentalization based treatment for borderline personality disorder. World Psychiatry, 9, 11-15.

Bateman, A., \& Fonagy, P. (2013). Impact of clinical severity on outcomes of mentalisation-based treatment for borderline personality disorder. British Journal of Psychiatry, 203, 221-227. doi:10.1192/bjp.bp.112.121129

Beecham, J., Sleed, M., Knapp, M., Chiesa, M., \& Drahorad, C. (2006). The cost and effectiveness of two psychosocial treatment programmes for severe personality disorder. European Psychiatry, 21, 102-109. doi:10.1016/j.eurpsy.2005.05.006

Bohus, M., Haaf, B., Simms, T., Limberger, M. F., Schmahl, C., Unckel, C., . . Linehan, M. M. (2004). Effectiveness of inpatient dialectical behavioral therapy for borderline personality disorder: a controlled trial. Behav Res Ther, 42, 487-499. doi: 10.1016/S0005-7967(03)00174-8 
Chiesa, M. (1997). A combined inpatient/outpatient programme for severe personality disorders. Therapeutic Communities, 18, 297-309.

Chiesa, M. (2005a). Can psychoanalytic research integrate and improve knowledge for the clinician? Some reflections and an example. The Scandinavian Psychoanalytic Review, 28, 31-39.

Chiesa, M. (2005b). Hospital-based psychosocial treatment of borderline personality disorder: A systematic review of rationale and evidence-base Clinical Neuropsychiatry, 2, 292-301.

Chiesa, M., Bateman, A., Wilberg, T., \& Friis, S. (2002). Patients' characteristics, outcome and cost-benefit of hospital-based treatment for patients with personality disorder: a comparison of three different programmes. Psychology and Psychotherapy, 75, 381-392.

Chiesa, M., \& Fonagy, P. (2000). Cassel personality disorder study: methodology and treatment effects. British Journal of Psychiatry, 176, 485-491.

Chiesa, M., \& Fonagy, P. (2002). From the therapeutic community to the community: A preliminary evaluation of a psychosocial outpatient service for severe personality disorders. Therapeutic Communities, 23, 247-259.

Chiesa, M., \& Fonagy, P. (2007). Prediction of medium-term outcome in cluster b personality disorder following residential and outpatient psychosocial treatment. Psychotherapy and Psychosomatics, 76, 347-353.

Chiesa, M., Fonagy, P., \& Gordon, J. (2009). Community-based psychodynamic treatment program for severe personality disorders: Clinical description and naturalistic evaluation. Journal of Psychiatric Practice, 15, 12-24. doi:10.1097/01.pra.0000344915.61706.d4

Chiesa, M., Fonagy, P., \& Holmes, J. (2003). When less is more: An exploration of psychoanalytically oriented hospital-based treatment for severe personality disorder. International Journal of Psycho-Analysis, 84, 637-650.

Chiesa, M., Fonagy, P., Holmes, J., \& Drahorad, C. (2004). Residential versus community treatment of personality disorder: A comparative study of three treatment programs. American Journal of Psychiatry, 161, 1463-1470.

Chiesa, M., \& Healy, K. (2009). The struggle to establish a research culture in the psychotherapy hospital: Reflections from the Cassel Hospital experience. Bulletin of the Menninger Clinic, 73, 157-174. doi:10.1521/bumc.2009.73.3.157

Chiesa, M., Sharp, R., \& Fonagy, P. (2011). Clinical associations of deliberate self-injury and its impact on the outcome of community-based and long-term inpatient treatment for personality disorder. Psychotherapy and Psychosomatics, 80, 100109. doi:10.1159/000320975

Clarkin, J. F., Levy, K. N., Lenzenweger, M. F., \& Kernberg, O. F. (2007). Evaluating Three Treatments for Borderline Personality Disorder: A Multiwave Study. American Journal of Psychiatry, 164, 922-928. doi:10.1176/appi.ajp.164.6.922

Crawford, M. J., Koldobsky, N., Mulder, R., \& Tyrer, P. (2011). Classifying personality disorder according to severity. J Pers Disord, 25, 321-330. doi:10.1521/pedi.2011.25.3.321

Davidson, K. M., \& Tran, C. F. (2014). Impact of treatment intensity on suicidal behavior and depression in borderline personality disorder: a critical review. J Pers Disord, 28, 181-197. doi:10.1521/pedi_2013_27_113

Davidson, K. M., Tyrer, P., Norrie, J., Palmer, S. J., \& Tyrer, H. (2010). Cognitive therapy $v$. usual treatment for borderline personality disorder: prospective 6-year follow-up. The British journal of psychiatry : the journal of mental science, 197, 456-462. doi:10.1192/bjp.bp.109.074286

De Panfilis, C., Politi, V., Fortunati, R., Cazzolla, R., Scaramuzzino, M., Marchesi, C., \& Maggini, C. (2011). Two-year follow-up of borderline personality disorder patients in Italy: a preliminary report on prognosis and prediction of outcome. The International journal of social psychiatry, 57, 528-537. doi: $10.1177 / 0020764010368619$

Ellis, P. D. (2010). The essential guide to effect sizes: Statistical power, meta-analysis, and the interpretation of research results: Cambridge University Press. 
Evans, C., Margison, F. R., \& Barkham, M. (1998). The contribution of reliable and clinically significant change methods to evidence-based mental health. Evidencebased Mental Health, 1, 70-72.

Fenton, W. S., \& McGlashan, T. H. (1990). Long-term residential care: Treatment of choice for refractory character disorder? Psychiatric Annals, 20, 44-49.

Field, A. (2013). Multilevel linear models. In A. Field (Ed.), Discovering Statistics Using IBM SPSS Statistics (4th ed., pp. 814-866). London: SAGE Publications.

First, M. B., Gibbon, M., Spitzer, R. L., Williams, J. B., \& Benjamin, L. S. (1997). Structured Clinical Interview for DSM-IV Axis-II Personality Disorders. Washington DC: American Psychiatric Press.

Fonagy, P., Luyten, P., \& Bateman, A. (2017). Treating Borderline Personality Disorder With Psychotherapy: Where Do We Go From Here? JAMA Psychiatry, 74, 316-317. doi: 10.1001/jamapsychiatry.2016.4302

Gabbard, G. O., Coyne, L., Allen, J. G., Spohn, H., Colson, D. B., \& Vary, M. (1999). Evaluation of intensive inpatient treatment of patients with severe personality disorders. Psychiatric Services, 51, 893-898.

Giesen-Bloo, J., van Dyck, R., Spinhoven, P., Van Tilburg, W., Dirksen, C., Van Asselt, T., ... Arntz, A. (2006). Outpatient Psychotherapy for Borderline Personality Disorder: Randomized Trial of Schema-Focused Therapy vs Transference-Focused Psychotherapy. Archives of General Psychiatry, 63, 649-659. doi:10.1001/archpsyc.63.6.649

Gowers, S. G., Weetman, A., Shore, A., Hossain, F., \& Elvins, R. (2000). Impact of hospitalisation on the outcome of adolescent anorexia nervosa. British Journal of Psychiatry, 176, 138-141.

Griffiths, P., \& Pringle, P. (Eds.). (1997). Psychosocial Practice within a Residential Setting. London: Karnac.

Gullestad, F. S., Wilberg, T., Klungsoyr, O., Johansen, M. S., Urnes, O., \& Karterud, S. (2012). Is treatment in a day hospital step-down program superior to outpatient individual psychotherapy for patients with personality disorders? 36 months follow-up of a randomized clinical trial comparing different treatment modalities. Psychother Res, 22, 426-441. doi:10.1080/10503307.2012.662608

Gunderson, J. G. (2001). Borderline Personality Disorder: A Clinical Guide. Washington, DC: American Psychiatric Publishing.

Hinshelwood, R. D., \& Skogstad, W. (1998). The hospital in the mind: In-patient psychotherapy at the Cassel Hospital. In J. Pestalozzi, S. Frisch, R. D. Hinshelwood, \& D. Houzel (Eds.), Psychoanalytic Psychotherapy in Institutional Settings (pp. 59-73). London: Karnac Books.

Horn, E. K., Verheul, R., Thunnissen, M., Delimon, J., Soons, M., Meerman, A. M., . . . Busschbach, J. J. (2015). Effectiveness of Short-Term Inpatient Psychotherapy Based on Transactional Analysis With Patients With Personality Disorders: A Matched Control Study Using Propensity Score. J Pers Disord, 29, 663-683. doi:10.1521/pedi_2014_28_166

Jacobson, N., \& Truax, P. (1991). Clinical significance: A statistical approach to defining meaningful change in psychotherapy research. Journal of Consulting and Clinical Psychology, 59, 12-19.

Leichsenring, F., Masuhr, O., Jaeger, U., Rabung, S., Dally, A., Dümpelmann, M., . . . Streeck, U. (2016). Psychoanalytic-Interactional Therapy versus Psychodynamic Therapy by Experts for Personality Disorders: A Randomized Controlled EfficacyEffectiveness Study in Cluster B Personality Disorders. Psychotherapy and Psychosomatics, 85, 71-80. doi:10.1159/000441731

Linehan, M. M., Comtois, K. A., Murray, A. M., Brown, M. Z., Gallop, R. J., Heard, H. L., . .. Lindenboim, N. (2006). Two-Year Randomized Controlled Trial and Follow-up of Dialectical Behavior Therapy vs Therapy by Experts for Suicidal Behaviors and Borderline Personality Disorder. Archives of General Psychiatry, 63, 757-766. doi:10.1001/archpsyc.63.7.757

National Institute for Health and Clinical Excellence. (2009). Borderline Personality Disorder: Treatment and Management. (CG78). London: Department of Health. 
Norton, K., \& Hinshelwood, R. D. (1996). Severe Personality Disorder: Treatment issues and selection for inpatient psychotherapy. British Journal of Psychiatry, 168, 723731.

Paris, J. (2004). Is hospitalization useful for suicidal patients with borderline personality disorder? J Personal Disord, 18, 240-247. doi:10.1521/pedi.18.3.240.35443

Prunetti, E., Bosio, V., Bateni, M., \& Liotti, G. (2013). Three-week inpatient Cognitive Evolutionary Therapy (CET) for patients with personality disorders: evidence of effectiveness in symptoms reduction and improved treatment adherence. Psychol Psychother, 86, 262-279. doi:10.1111/j.2044-8341.2011.02060.x

Rabe-Hesketh, S., \& Skrondal, A. (2008). Multilevel and longitudinal modeling using STATA. College Station, Texas: Stata Press.

Rutter, D., \& Tyrer, P. (2003). The value of therapeutic communities in the treatment of personality disorder: A suitable place for treatment? Journal of Psychiatric Practice, 9, 291-302.

Schimmel, P. (1997). Swimming against the tide? A review of the therapeutic community. Australian and New Zealand Journal of Psychiatry, 31, 120-127.

Siefert, C. J. (2012). A goal-oriented limited-duration approach for borderline personality disorder during brief inpatient hospitalizations. Psychotherapy (Chic), 49, 502518. doi: $10.1037 / \mathrm{a} 0026128$

Tyrer, P., Reed, G. M., \& Crawford, M. J. (2015). Classification, assessment, prevalence, and effect of personality disorder. The Lancet, 385, 717-726. doi:10.1016/S01406736(14)61995-4

Vermote, R., Luyten, P., Verhaest, Y., Vandeneede, B., Vertommen, H., \& Lowyck, B. (2015). A psychoanalytically informed hospitalization-based treatment of personality disorders. Int J Psychoanal, 96, 817-843. doi:10.1111/17458315.12394

Zanarini, M. C. (2009). Psychotherapy of borderline personality disorder. Acta Psychiatr Scand, 120, 373-377. doi:10.1111/j.1600-0447.2009.01448.x

Zanarini, M. C., Frankenburg, F. R., Hennen, J., Reich, D., \& Silk, K. R. (2006). Prediction of the 10-year course of borderline personality disorder. American Journal of Psychiatry, 163, 827-832. doi:10.1176/ajp.2006.163.5.827

Zanarini, M. C., Frankenburg, F. R., Hennen, J., Reich, D. B., \& Silk, K. R. (2005). The McLean Study of Adult Development (MSAD): overview and implications of the first six years of prospective follow-up. J Personal Disord, 19, 505-523.

Zanarini, M. C., Frankenburg, F. R., Reich, D. B., Wedig, M. M., Conkey, L. C., \& Fitzmaurice, G. M. (2014). Prediction of time-to-attainment of recovery for borderline patients followed prospectively for 16 years. Acta Psychiatr Scand, 130, 205-213. doi:10.1111/acps. 12255 